\title{
Rapid fabrication of polymer microfluidic systems for the production of artificial lipid bilayers
}

\author{
M E Sandison and H Morgan \\ School of Electronics and Computing Science, University of Southampton, Southampton, \\ SO17 1BJ, UK \\ E-mail: mes@ecs.soton.ac.uk and hm@ecs.soton.ac.uk
}

Received 6 January 2005, in final form 22 February 2005

Published 20 June 2005

Online at stacks.iop.org/JMM/15/S139

\begin{abstract}
A polymer microfluidic device has been fabricated using rapid prototyping techniques. The device was built to allow the formation and subsequent investigation of artificial bilayer lipid membranes (BLMs). A simple dry film photoresist stamp was used to hot emboss microfluidic channels into PMMA films. Laser micromachining was employed to form an aperture through the PMMA channels, across which the BLM was later formed. The dry film photoresist was also used as a simple etch mask for the deep etching of glass substrates in buffered HF solutions, which was used in this work for the production of glass embossing stamps. We show that bilayer films can be successfully produced across laser micromachined apertures in PMMA films.
\end{abstract}

\section{Introduction}

Microfabricated devices for the formation of artificial bilayer lipid membranes (BLMs) could have widespread applications in a number of areas. These include the production of ultra-sensitive single molecule biosensors, drug screening technologies and the creation of model biological systems for fundamental biophysical studies. BLMs are produced when amphipathic lipid molecules spontaneously self-assemble into a thermodynamically favourable bilayer configuration. The membrane is formed from two monolayer sheets of phospholipid molecules with the hydrophobic, hydrocarbon chains of the molecules on the inside and the hydrophilic, negatively charged head groups on the outside (Tien and Ottawa 2001). Many drug targets are membrane proteins and these proteins can be reconstituted into BLMs by a variety of methods, including the fusion of protein-containing lipid vesicles (Woodbury and Miller 1990). Therefore, the development of new analytical microsystems based upon artificial bilayer membranes would have many uses in drug screening technology (Bayley and Cremer 2001).

A variety of lipid bilayer assemblies have been produced over the years, including solid supported and gel supported BLMs (Trojanowicz and Mulchandani 2004), BLMs formed over nanoporous structures (Favero et al 2002, Römer and Steinem 2004) and suspended BLMs, formed across apertures in a thin sheet of a hydrophobic material (Montal and Mueller 1972). BLMs are typically formed across an aperture with a diameter in the range of $10-100 \mu \mathrm{m}$ that is mounted between two large electrolyte reservoirs. This provides an environment for reconstituted proteins that is similar to their native one, with the bilayer surrounded on both sides by water. However, this traditional apparatus is not amenable to high resolution optical studies of membrane proteins or to high throughput BLM investigations. In contrast, microfluidic systems could be employed to satisfy both these requirements.

To address these issues, there has been increasing interest in developing microfabricated devices for supporting and studying BLMs. Such devices have been produced with apertures in silicon (Osborn and Yager 1995), glass (Fertig et al 2001) and silicon nitride (Morgan et al 2004, Peterman et al 2002). Although numerous reproducible, well-defined apertures can be produced in silicon-based materials using standard microfabrication techniques, silicon does have some major disadvantages. Native silicon has a very low resistance compared with the bilayer (which is in the $G \Omega$ range) so that large background currents can occur. In the case of insulated silicon supports, there is often a large shunt capacitance across the bilayer, due to the use of thin dielectric films, leading to increased electrical noise and restricted bandwidth, whilst silicon nitride membranes themselves are extremely fragile. Furthermore, the fabrication route is time consuming, 


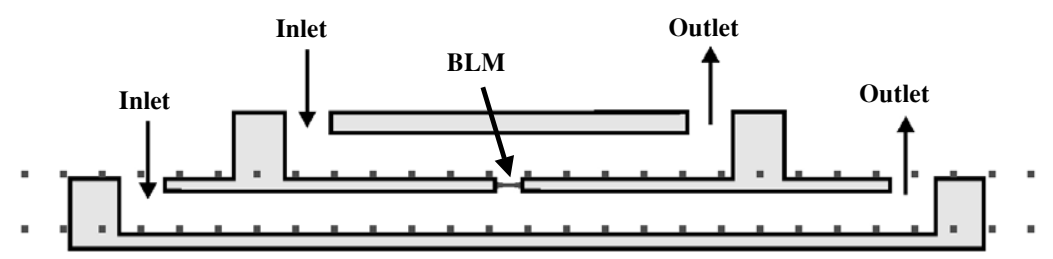

Figure 1. Schematic illustrating a cross-section of the polymer microfluidic system for artificial BLM formation. The system comprises upper and lower fluidic channels that are separated by a single aperture, where the artificial BLM will be formed.

expensive and requires clean room facilities. Alternative methods have been described (Mayer et al 2003), involving the application of soft lithography techniques. By casting Teflon $\mathrm{AF}^{\circledR}$ solution over PDMS moulds, these authors were able to manufacture well-defined apertures, which were then used in a traditional BLM experimental system.

In this paper, we describe an alternative approach to the fabrication of BLM supports that is based on the use of polymer rapid fabrication techniques (Becker and Gärtner 2000). This provides a simple, low-cost alternative to the production of microfabricated devices for the formation of artificial BLM systems and also eliminates many of the drawbacks of siliconbased devices. The rapid fabrication of such polymeric devices should also be of use in a wide variety of microfluidic applications.

Multi-layered microfluidic devices were fabricated rapidly and simply by a combination of hot embossing and thermal or solvent bonding. A central aperture for supporting the BLM was formed using laser micromachining. (Traditionally apertures for supporting BLMs are produced in the thin polymer films using hot needles. Compared to this method, laser micromachining can produce apertures with a well-defined geometry at a precise location in a sample.) These microfluidic devices will allow for the rapid exchange of electrolytes and the injection of small quantities of analytes, which will minimize the quantities of reagents required and will promote rapid transport of material to and from the artificial BLM. A schematic diagram of the device is presented in figure 1. The device is formed from three polymer films bonded together: an unstructured base plate (beneath the lower dotted line in figure 1); a polymer film with a hot embossed microfluidic channel that has an aperture for BLM formation laser micromachined through the base (between the dotted lines) and a second polymer film with a hot embossed upper microfluidic channel (above the upper dotted line). The device is mounted over a microscope and can therefore be used for the simultaneous acquisition of both electrical signals (conductance and capacitance) and optical measurements (generally single molecule fluorescence).

In order to hot emboss the polymer films, dry film photoresist stamps were employed, which were rapidly and simply fabricated without the use of cleanroom facilities. These photoresist layers also proved effective as an etch mask for deep glass etching, which enabled us to produce embossing stamps made from glass without having to employ more complicated multi-layer masks. Although the production of shallow etched feature using thin resist masks is relatively straightforward, the production of deep etched structures is notoriously difficult because most masking materials are either attacked by the HF, detach from the substrate or contain pinholes. To solve these problems, multilayers of metal $(\mathrm{Cr} / \mathrm{Au} / \mathrm{Cr} / \mathrm{Au})$ in combination with thick photoresist films have been employed ( $\mathrm{Bu}$ et al 2004). In comparison, dry film photoresists offer a very simple, effective alternative etch mask.

\section{Experimental section}

\section{Materials}

All reagents were obtained from Sigma-Aldrich (Poole, UK), unless otherwise stated, and all polymer films were purchased from Goodfellow (Cambridge, UK). The dry film photoresists employed in this work were Ordyl Alpha 920 and Ordyl AM 175, both obtained from Elga Europe (Daventry, UK). Glass microscope slides $(52 \times 75 \times 1 \mathrm{~mm})$ were purchased from VWR (Leicestershire, UK), and Macor ${ }^{\circledR}$ ceramic plates were obtained from RS Components (Corby, UK). Ecoclear cleaning fluid was purchased from Logitech (Glasgow, UK). The buffered oxide etchant (BOE) used for etching glass substrates was a 7:1 (40\% $\left.\mathrm{NH}_{4} \mathrm{~F}: 49 \% \mathrm{HF}\right)$ silicon dioxide etchant (Rockwood Electronic Materials, Derbyshire, UK). Lipid bilayers were formed using 1,2-diphytanoyl-sn-glycero3-phosphocholine (Avanti Polar Lipids Alabama, USA).

\section{Microfabrication}

Fabrication of embossing stamps. Embossing stamps were produced using the negative tone, dry film photoresists Ordyl Alpha 920 (a $20 \mu \mathrm{m}$ thick film) and Ordyl AM 175 (a $75 \mu \mathrm{m}$ thick film). The former was processed as follows. Glass microscope slides were cleaned by ultrasonicating at $60{ }^{\circ} \mathrm{C}$ for $10 \mathrm{~min}$ in Ecoclear, then acetone and finally in methanol. The sample was then thoroughly rinsed with deionized water. Before lamination, the slide was dehydrated by baking at 200 ${ }^{\circ} \mathrm{C}$ for $1 \mathrm{~h}$ and the surface was subsequently primed by spincoating a film of hexamethyldisilane (HMDS) at $3000 \mathrm{rpm}$ for $20 \mathrm{~s}$. The dry film resist was then laminated onto the substrate using a Mega Electronics A4 laminator (Cambridge, UK) heated to $115{ }^{\circ} \mathrm{C}$, with a speed of $0.15 \mathrm{~m} \mathrm{~min}^{-1}$. To improve the adhesion of the resist, a $10 \mathrm{~min}$ pre-exposure bake was performed at $120{ }^{\circ} \mathrm{C}$. A Mega Electronics vacuum UV exposure unit, equipped with $6 \times 15 \mathrm{~W}$ bulbs, was then used to expose the sample for $40 \mathrm{~s}$ through a lowcost polyester film mask (J.D. Photo-Tools, Oldham, UK). The sample was developed for approximately $90 \mathrm{~s}$ in an ultrasonic bath using a $0.8 \%(\mathrm{w} / \mathrm{v})$ sodium carbonate solution and was then rinsed thoroughly with deionized water. The sample was subsequently hard baked at $195{ }^{\circ} \mathrm{C}$ for $30 \mathrm{~min}$, ramping the temperature up from room temperature, at a rate 

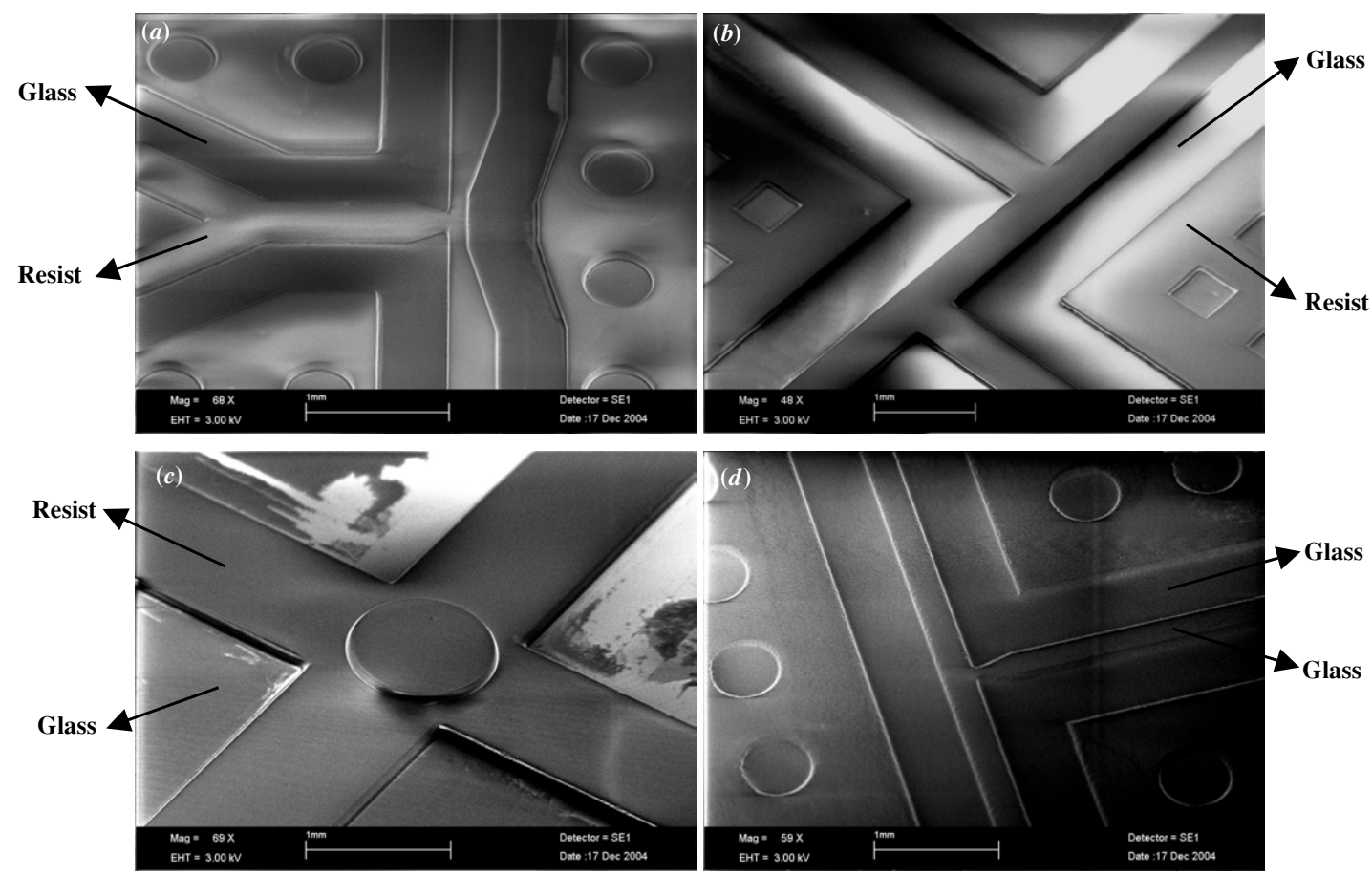

Figure 2. Dry film photoresist and glass embossing stamps. (a) An embossing stamp made from AM 175 dry film resist. (b) A stamp produced using four layers of Alpha 920. (c) A stamp with two individually patterned AM 175 layers. (d) A wet etched glass stamp (the features in this sample are approximately $45 \mu \mathrm{m}$ deep). The bright areas in regions of these SEMs result from charging of the sample. For clarity, regions of dry film resist and of glass substrate are labelled.

of approximately $5{ }^{\circ} \mathrm{C} \mathrm{min}^{-1}$, and allowing the oven to cool to below $50^{\circ} \mathrm{C}$ before removing the sample.

The $75 \mu \mathrm{m}$ resist (AM 175) was processed similarly. However, as this resist formulation contains significantly more primer, the need for an adhesion promoter was obviated, so the HMDS primer and the pre-exposure bake were no longer employed. This thicker resist was developed in $2 \%(\mathrm{w} / \mathrm{v})$ sodium carbonate solution.

At temperatures in excess of $150-160{ }^{\circ} \mathrm{C}$, it was observed that the laminate resist begins to soften and becomes slightly tacky. Therefore, the dry film resist structures were only used to hot emboss polymers whose glass transition temperature $\left(T_{\mathrm{g}}\right)$ was lower than $140{ }^{\circ} \mathrm{C}$. For processing polymers with higher $T_{\mathrm{g}}$ values, the dry film resist was used as a mask to produce a glass embossing stamp by wet chemical etching. The dry film resist mask was prepared as described previously. In addition, the glass microscope slides were annealed before use at $400{ }^{\circ} \mathrm{C}$ to reduce the residual stress within the glass (Lin et al 2001). Glass etching was carried out in a continuously stirred, $50 \%$ BOE, $10 \%$ concentrated hydrochloric acid bath (the addition of hydrochloric acid was used to prevent the formation of insoluble crystalline precipitates (Lin et al 2001)). With this solution, the etch rate was approximately $2.2 \mu \mathrm{m} \mathrm{min}^{-1}$.

Hot embossing and bonding of PMMA films. Poly(methyl methacrylate) (PMMA) films were embossed by clamping a piece of polymer between the microfabricated stamp and a polished Macor ceramic plate using a conventional G-clamp. This assembly was placed in an oven, heated to $135^{\circ} \mathrm{C}$ (the glass transition temperature of PMMA being $115^{\circ} \mathrm{C}$ ) and held at this temperature for $30 \mathrm{~min}$. Before removing the clamp, the assembly was left to cool to room temperature. Other polymeric materials were also embossed using the same procedure. These included poly(ethylene terephthalate) (PET), embossed at $115^{\circ} \mathrm{C}$, poly(tetrafluoroethylene) (PTFE), embossed at $350{ }^{\circ} \mathrm{C}$ and fluorinated ethylene propylene copolymer (FEP), embossed at $250{ }^{\circ} \mathrm{C}$. The later two were embossed using a glass stamp, whilst PET and PMMA were embossed using a dry film resist stamp.

Following embossing, the PMMA films were aligned and bonded using both solvent (Wang et al 2002) and thermal bonding methods. In the case of the former, the surface of an embossed sample and a base plate were first treated using a reactive ion etching machine with an oxygen plasma (5 min at $100 \mathrm{~W}, 32 \mathrm{mT}$ and $30 \mathrm{SCCM} \mathrm{O}_{2}$ ). The surface of the base plate was subsequently flooded with acetyl acetone and, after waiting for approximately $5 \mathrm{~s}$, was spun at $4000 \mathrm{rpm}$ for $2 \mathrm{~s}$. The sample was then immediately placed over the base plate and, using a glass microscope slide, was manually pressed for a minimum of $2 \mathrm{~min}$. In the case of thermal bonding, two embossed pieces of PMMA were aligned using an inspection microscope and then clamped between two polished ceramic plates. This assembly was then baked in an oven at $110{ }^{\circ} \mathrm{C}$ for $30 \mathrm{~min}$. The bonded sample was left to cool to room temperature before the clamp was removed.

Laser micromachining of apertures. Samples of PMMA, PTFE, PET and FEP were processed at the Laser Micromachining Centre in the Institute for Bioelectronics and Molecular Microsystems, University of Wales, Bangor using an Exitech laser micromachining tool equipped with a $280 \mathrm{~nm}$ 

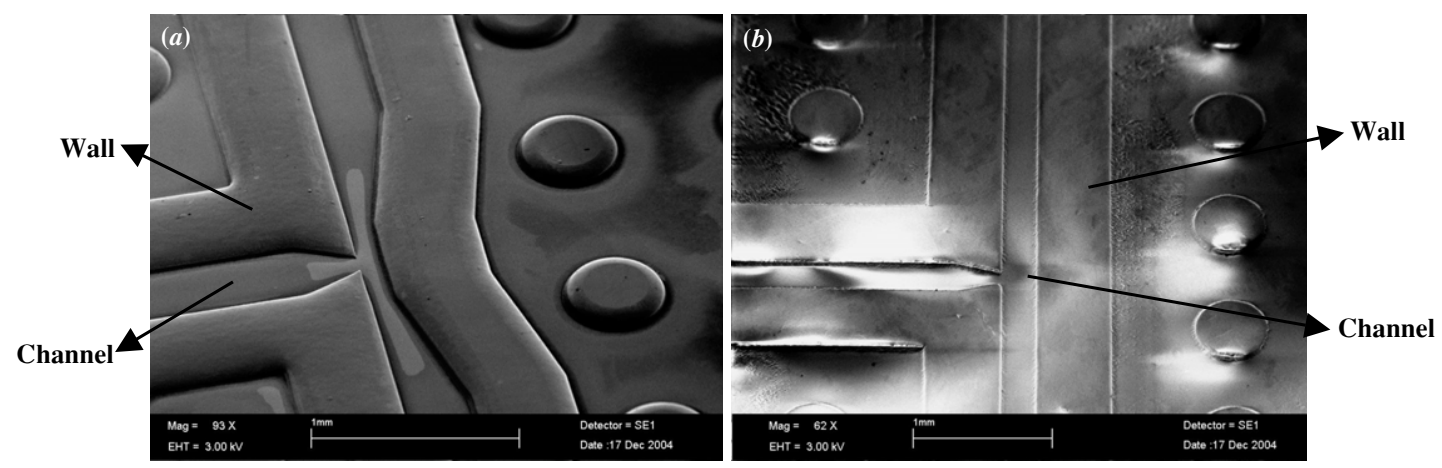

Figure 3. Hot embossed polymer films. SEMs showing embossed PMMA $(a)$ and PTFE $(b)$ films. Again, the bright areas in regions of these SEMs result from charging of the sample. For clarity, the microfluidic channels and channel walls are labelled.

Eximer laser, operating with a pulse width of $130 \mathrm{fs}$ and focal spot size of approximately $20 \mu \mathrm{m}$.

\section{Experimental methods}

In order to investigate the use of the various micromachined polymer films, BLMs were formed across apertures in all the polymers using the conventional Montal-Mueller technique (Montal and Mueller 1972, Mayer et al 2003). Bilayers were formed in the microfluidic devices using the following method. The lipid 1,2-diphytanoyl-sn-glycero-3-phosphocholine was diluted with n-decane, to a concentration of $1 \mathrm{mg} \mathrm{ml}^{-1}$. A plug of this lipid (in the order of $5 \mu \mathrm{l}$ ) was passed through both the upper and lower fluidic channels. First the lower channel and secondly the upper channel were then slowly filled with the electrolyte solution $(1 \mathrm{M} \mathrm{KCl})$. BLM formation, which was not always observed after injection of the electrolyte, occurred by spontaneous thinning of the lipid remaining within the aperture. In the cases where a lipid bilayer did not form within a few minutes of adding the electrolyte, the fluid in either of the buffer channels was removed and then re-injected, so as to encourage the thinning process. BLM formation was monitored electrically using $\mathrm{Ag} / \mathrm{AgCl}$ electrodes inserted into the upper and lower buffer streams. These were connected to a high sensitivity ID562 BLM amplifier (Industrial Developments Bangor, Bangor, UK). Optical monitoring of the bilayer was performed using a homemade microscope, based around a Nikon $\times 40$ objective lens with a $720 \mu \mathrm{m}$ working distance, a $532 \mathrm{~nm}$ frequency doubled YAG laser and an iXon electron-multiplying CCD camera (Andor Technology, Belfast, Northern Ireland).

\section{Results and discussion}

\section{Microfabrication of polymer devices}

A hot embossing stamp produced using Ordyl AM 175 dry film photoresist is shown in the SEM of figure 2(a) (all samples were coated with approximately 10-20 nm gold before observation in a Leo 430 SEM). As dry film photoresists are available in a range of thickness (between 20 and $100 \mu \mathrm{m}$ ) and multiple resist layers can be laminated onto one sample (such as the example shown in figure $2(b)$ ), stamps with a wide variety of geometries can be produced. This includes stamps with two or more steps in height, a simple example of which is shown in figure $2(c)$. This was fabricated by sequentially patterning two dry film resist layers, the lower cross-shaped channel being processed as described previously before a second resist layer was laminated and patterned to produce the upper circle.

If the dry film resist is hard baked at $195{ }^{\circ} \mathrm{C}$, it becomes very robust, although some shrinkage will occur (approximately 3\% in the case of AM 175 and 15\% in the case of Alpha 920). Hard-baked resist stamps were used numerous times without any degradation of the structure and they could withstand ultrasonic cleaning in detergent solutions, alcohols and acetone. Additionally, the hard-baked resist could be stripped from a substrate by immersion in fuming nitric acid.

On occasion, removal of the embossed polymer films from the AM 175 stamps proved to be problematic, presumably because this photoresist is formulated to adhere strongly to a wide variety of substrates. No such problems were encountered when using the Alpha 920 stamps. To determine the minimum feature size that could be obtained when using a simple (non-collimated) UV exposure unit with the Alpha 920 photoresist, a range of pillars with different diameters were fabricated. It was found that $25 \mu \mathrm{m}$ diameter pillars could be resolved (although, due to the non-vertical side walls obtained, the footprint at the base of the pillars was somewhat wider than this). It is expected that this resolution could be improved significantly using a collimated light source with a standard mask alligner (Vulto et al 2004).

When processed as outlined above, both dry film photoresists proved to be effective etch masks for use in buffered HF solutions, enabling deep etching of glass substrates. The initial annealing and dehydration step and, in the case of Alpha 920, the use of the HMDS primer were essential for good adhesion of the photoresist to the glass surface, which is critical to the performance of an etch mask. Glass stamps with features $10-150 \mu \mathrm{m}$ deep have been produced, without any noticeable degradation of the mask. Presumably, greater etch depths should be possible. Figure $2(d)$ shows a glass stamp (approximately $45 \mu$ m deep) produced using an Alpha 920 etch mask.

A PMMA film embossed using the AM 175 stamp of figure 2(a) is shown in figure $3(a)$. It can be seen that the features in the original sample are faithfully reproduced in the PMMA film. For polymers with high $T_{\mathrm{g}}$ values, such as PTFE, glass stamps were used for embossing. An example of this is shown in figure $3(b)$, where a film of PTFE was successfully embossed using the stamp shown in figure $2(d)$. 

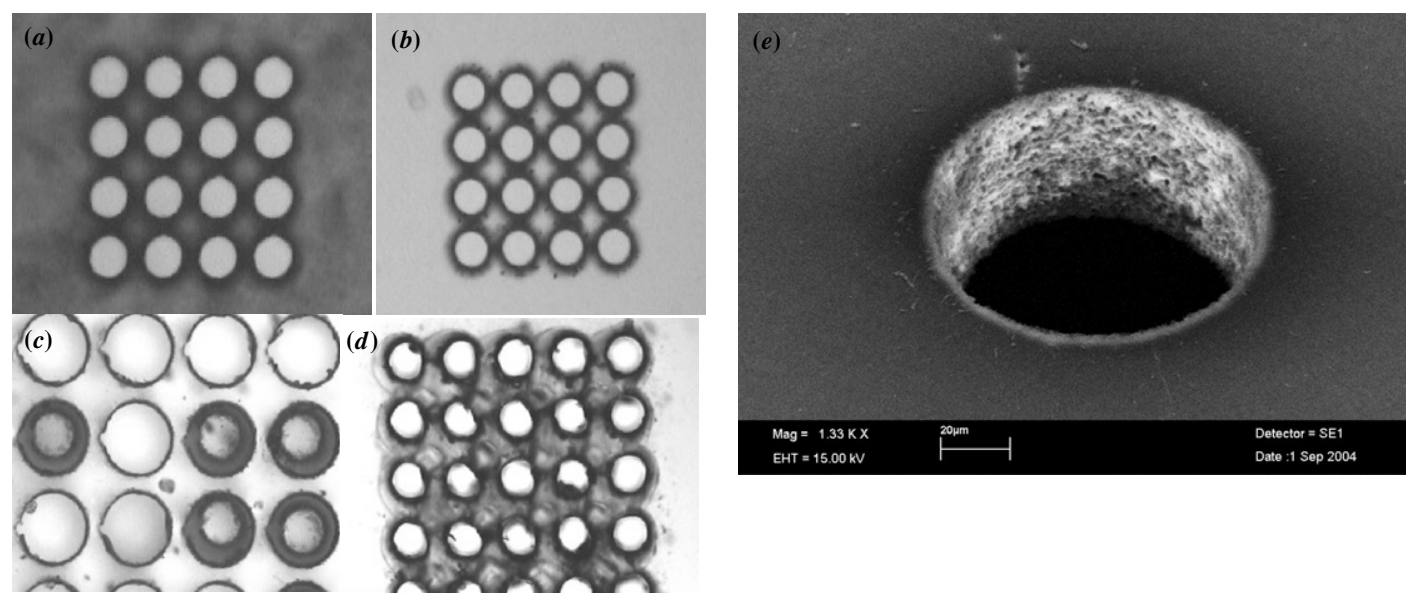

Figure 4. Laser micromachined apertures. (a) $50 \mu \mathrm{m}$ diameter apertures in a $50 \mu \mathrm{m}$ thick PTFE film. (b) $50 \mu \mathrm{m}$ apertures in $50 \mu \mathrm{m}$ thick PMMA. (c) $100 \mu \mathrm{m}$ apertures in $250 \mu \mathrm{m}$ thick FEP. $(d) 100 \mu \mathrm{m}$ apertures in $250 \mu \mathrm{m}$ thick PET. (e) A $100 \mu \mathrm{m}$ diameter aperture machined through a PMMA film.
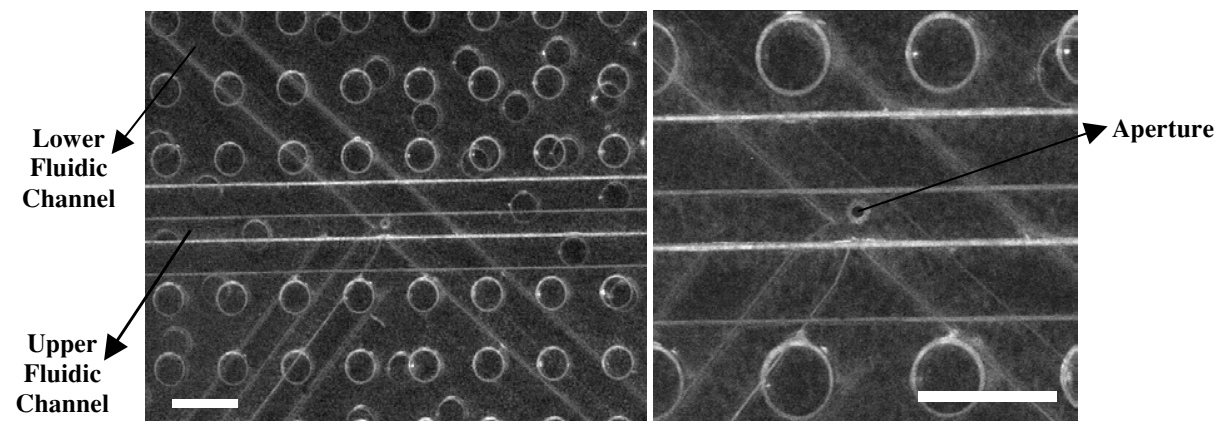

Figure 5. Double-channel microfluidic device. A photograph of a microfluidic device with a T-channel beneath a micromachined aperture and a straight channel above. The channels are formed using $500 \mu \mathrm{m}$ wide channel walls with an array of supporting pillars. The scale bar represents $1 \mathrm{~mm}$.
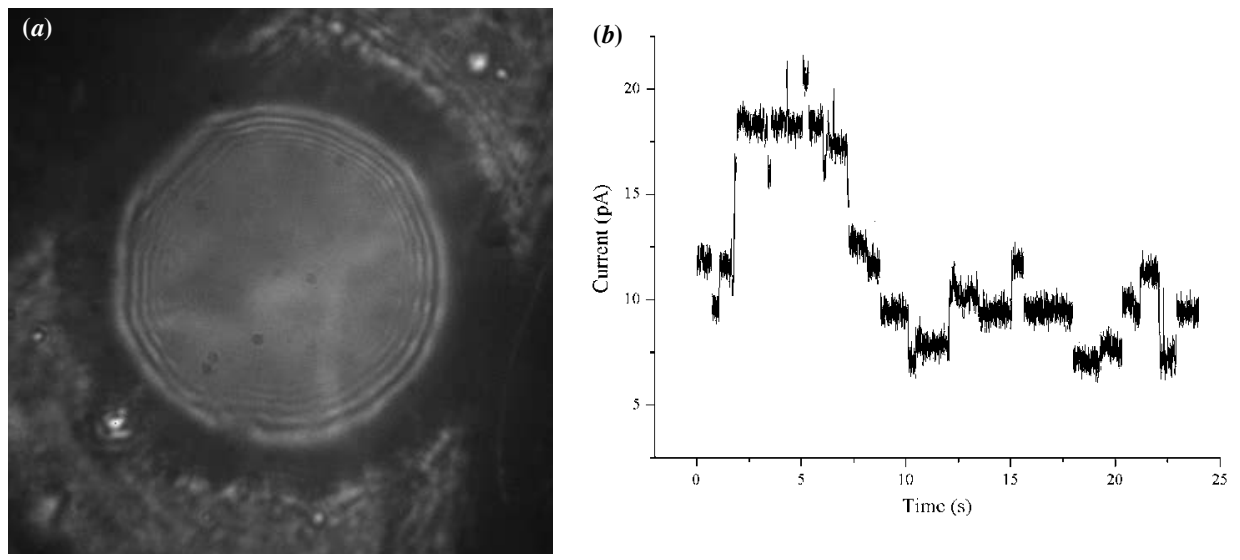

Figure 6. BLM formation across a PMMA aperture. (a) A reflectance microscopy image of the thinning of a lipid film across a $100 \mu \mathrm{m}$ aperture in a PMMA microfluidic device. (b) A current-time trace that shows ion conduction through single gramicidin dimmer ion-channels, which form temporarily when two gramicidin monomers come together by diffusion. Data were acquired in a $1 \mathrm{M} \mathrm{KCl}$ solution at a potential of $100 \mathrm{mV}$.

The microfluidic structures were made from two layers of embossed material, but prior to construction a 50-100 $\mu \mathrm{m}$ diameter aperture was created in one of the polymer films by laser micromachining. Preliminary tests showed that the arrays of high quality apertures could be made relatively easily in both PMMA and PTFE films, but that significant optimization may be required to successfully machine the FEP and PET films. The results of these laser micromachining tests are shown in figures $4(a)-(d)$. In the case of the FEP, although some apertures were successfully machined, others remained partially blocked; whilst for the PET sample, the heat generated by the laser resulted in the deformation of the material surrounding the apertures. In view of these results, PMMA was selected as the optimum material for fabrication of 
the microfluidic devices, as it could be machined with relative ease, its chemical resistance is satisfactory, its embossing temperature is relatively low and it is a transparent material with optical properties similar to that of glass (its refractive index is 1.49 compared to 1.51 for glass and they have an equal transmission of visible light of $92 \%$ ). A SEM of a clean, welldefined aperture laser micromachined through a PMMA film is shown in figure 4(e). Though the walls of the aperture are clearly rough, it is presumed that this will not have a significant effect on the formation or the quality of BLMs - traditional apertures formed using a hot needle are also somewhat rough, yet robust, low-noise BLMs can be routinely formed across them.

Embossed PMMA films were successfully bonded using both thermal and solvent bonding. Thermally bonding two films under pressure at a temperature slightly below the $T_{\mathrm{g}}$ of PMMA was found to be the simplest of the two methods, as this technique does not require an oxygen plasma etcher. However, fluctuations in oven temperature and variation in applied pressure can noticeably affect the final device. After optimization of the process, solvent bonding proved to be highly reproducible, but as the two PMMA films were manually assembled immediately after spin-coating, little or no alignment was possible. Therefore, this method was employed only for bonding embossed films to non-structured base plates. A completed microfluidic device, bonded together using thermal bonding, is shown in figure 5 .

\section{Formation of BLMs}

Using the traditional Montal-Mueller technique, it was found that stable, noise-free bilayers could be formed on all the polymers investigated. BLMs were also successfully produced across a laser micromachined aperture within a microfluidic device. BLM formation was monitored by recording the increase in membrane capacitance and by observing the outward moving interference fringes (that were seen using the microscope in reflection mode) as thinning of the lipid film occurred over time. Such interference fringes are shown in figure $6(a)$. Gramicidin ion-channels were reconstituted into a BLM formed across a micromachined PMMA aperture. The current-time trace shown in figure $6(b)$ is representative of the behaviour of the trans-membrane peptide. These data confirm that a bilayer had successfully formed and that low-noise, single channel recordings can be obtained. Furthermore, these results suggest that the quality of BLMs produced in a polymer microfluidic system should be comparable to results obtained using alternative BLM formation techniques, with high quality seals and stable BLMs being obtainable.

\section{Conclusion}

A rapid and simple fabrication protocol for producing microfluidic systems has been developed. Robust stamps for the hot embossing of polymers such as PMMA were produced using a dry film photoresist, which also proved to be an effective etch mask for wet chemical etching of glass. The microfluidic devices produced will enable the simultaneous optical and electrical observation of artificial BLMs, which were successfully produced within the microfluidic device.

\section{Acknowledgments}

The authors would like to acknowledge Dr Nadeem Rizvi (University of Wales, Bangor) for carrying out the laser micromachining work and to thank John Homer of Elga Europe for helpful discussions. This work was supported by the UK Interdisciplinary Research Centre in BioNanotechnology (R45659/01).

\section{References}

Bayley H and Cremer P S 2001 Stochastic sensors inspired by biology Nature 143 226-30

Becker H and Gärtner C 2000 Polymer microfabrication methods for microfluidic analytical applications Electrophoresis 21 12-26

Bu M et al 2004 A new masking technology for deep glass etching and its microfluidic application Sensors Actuators A 115 476-82

Favero G et al 2002 Membrane supported bilayer lipid membranes array: preparation, stability and ion-channel insertion Anal. Chim. Acta 460 23-34

Fertig N et al 2001 Microstructured glass chip for ion-channel electrophysiology Phys. Rev. E 64040901

Lin C-H et al 2001 A fast prototyping process for fabrication of microfluidic systems on soda-lime glass J. Micromech. Microeng. 11 726-32

Mayer M et al 2003 Microfabricated Teflon membranes for low-noise recordings of ion-channels in planar lipid bilayers Biophys. J. 85 2684-95

Montal M and Mueller P 1972 Formation of bimolecular membranes from monolayers and study of their electrical properties Proc. Natl Acad. Sci. USA 69 3561-6

Morgan $\mathrm{H}$ et al 2004 Artificial lipid bilayers in a microfabricated system Proc. $\mu$ TAS 2004 vol 1 ed T Laurell et al pp 330-2

Osborn T D and Yager P 1995 Formation of planar solvent-free phospholipid bilayers by Langmuir-Blodgett transfer of monolayer to micromachined apertures in silicon Langmuir $118-12$

Peterman M C et al 2002 Ion channels and lipid bilayer membranes under high potentials using microfabricated apertures Biomed. Microdevices 4 231-6

Römer W and Steinem C 2004 Impedance analysis and single channel recordings on nano-black lipid membranes based on porous alumina Biophys. J. 86 955-65

Tien H T and Ottova A L 2001 The lipid bilayer concept and its experimental realization: from soap bubbles, kitchen sink to bilayer lipid membranes J. Membrane Sci. 189 83-117

Trojanowicz M and Mulchandani A 2004 Analytical applications of planar bilayer lipid membranes Anal. Bioanal. Chem. 379 347-50

Vulto P et al 2004 Dry film resist for fast fluidic protyping Proc. $\mu$ TAS 2004 vol 2 ed T Laurell et al pp 43-5

Wang J et al 2002 Towards disposable lab-on-a-chip: poly(methylmethacrylate) microchip electrophoresis device with electrochemical detection Electrophoresis 23 596-601

Woodbury D J and Miller C 1990 Nystatin-induced liposome fusion Biophys. J. 58 833-9 